\title{
Influence of manufacturing defects on the mechanical behaviour of all-composite wing under service load conditions.
}

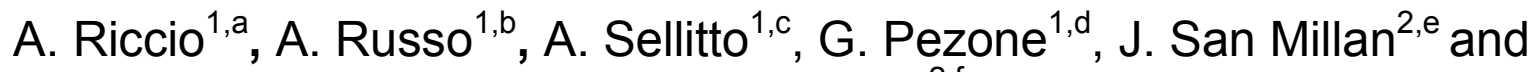 \\ B. I. Armendariz ${ }^{2, f}$ \\ ${ }^{1}$ Department of Industrial and Informatics Engineering, Università della Campania "Luigi \\ Vanvitelli", via Roma 29, 81031 Aversa (CE), Italy \\ ${ }^{2}$ INTA - Departamento de Materiales y Estructuras, Carretera de Ajalvir km 4 \\ 28850 Torrejón de Ardoz - Madrid, Spain \\ aaniello.riccio@unicampania.it, bangela.russo@unicampania.it, \\ candrea.sellitto@unicampania.it, 'gppezone@gmail.com, esanmillan@inta.es,
}

Keywords: Manufacturing defects; Delamination; Damage tolerance; Buckling.

\begin{abstract}
In this paper, the damage behaviour of aerospace composite structures has been studied. The analysed structure is an all-composite wing with inter-laminar manufacturing induced damage. The manufacturing induced delaminations are located at the leading edge on the extrados and in the area near the fuselage. Different analyses have been performed to correctly predict the pre-existent damages evolution under service loading conditions. Preliminary linear buckling analyses have been performed to check for instability of the delaminations under service loads; then the risk of delaminations' propagation has been evaluated by means of a Virtual Crack Closure Technique based approach.
\end{abstract}

\section{Introduction}

Progress in aerospace field strongly depends on the development of lightweight structures which are resistant to mechanical, thermal and environmental loads. For this reason, the composite materials are widely used for aerospace structures manufacturing. Indeed, their excellent mechanical properties in terms of strength-weight and stiffness-weight ratios, allow the weight reduction of the structures if compared conventional metallic alloys. On the other hand, composite materials are strongly vulnerable to damage. One of the most common failure modes in composite structures is the delamination, which consist in the separation between two or more adjacent layers of a laminate able to reduce the carrying load capability of the structures. Delamination, arising as a consequence of impact with foreign objects [1-4], applied loads or manufacturing processes [5-6], has been widely investigated, both numerically and experimentally, in literature. Indeed, in this paper, an allcomposite wing with manufacturing defects, such as delaminations occurring on the wing's extrados and close to the front spar, has been investigated. Linearized buckling and non-linear numerical analyses have been performed to predict the manufacturing induced damages elastic instability and evolution under service loading conditions.

\section{Numerical Model}

The wing of an UAV (Unmanned Aerial Vehicle), developed and realised at the INTA (Instituto Nacional de Técnica Aeroespacial) facilities, has been numerically investigated. The wing has been entirely realised by using composite laminates made of two composite materials systems, whose properties are listed in Table 1. 


\begin{tabular}{|c|c|c|}
\cline { 2 - 3 } \multicolumn{1}{c|}{} & $\begin{array}{c}\text { UNIDIRECTIONAL } \\
\text { IM7/MTM45- }\end{array}$ & $\begin{array}{c}\text { TEXTILE LAYER 0/90 MTM45- } \\
\text { 1/CF5542-40\% RW }\end{array}$ \\
\hline $\mathrm{E}_{1}[\mathrm{MPa}]$ & 157000 & 69000 \\
\hline $\mathrm{E}_{2}[\mathrm{MPa}]$ & 8500 & 69000 \\
\hline $\mathrm{E}_{3}[\mathrm{MPa}]$ & 8500 & 8500 \\
\hline $\mathrm{G}_{12}[\mathrm{MPa}]$ & 3890 & 3400 \\
\hline $\mathrm{G}_{23}[\mathrm{MPa}]$ & 3890 & 3400 \\
\hline $\mathrm{G}_{31}[\mathrm{MPa}]$ & 3890 & 3400 \\
\hline$v_{12}=v_{13}$ & 0.34 & 0.0375 \\
\hline$v_{23}$ & 0.0925 & 0.17 \\
\hline & Table 1. Materials properties.
\end{tabular}

As already mentioned, during the manufacturing process, several delaminations occurred at different locations. The shapes and the depths of the defects have been evaluated performing ultrasonic inspections as reported in Fig. 1.

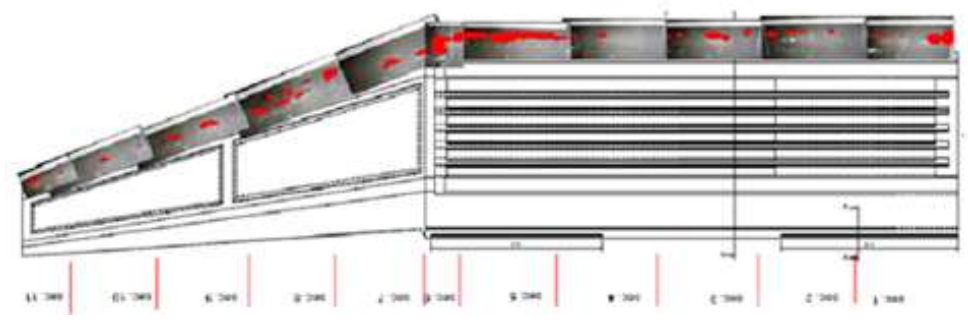

Fig. 1. Ultrasonic inspection.

The finite elements model of the wing has been realised by adopting the FE software ABAQUS and discretized by using four-node shell elements (S4). On the other hand, the wing zone, in which the delaminations are located, has been modelled by using eight-node brick element with a reduced integration scheme (C3D8R). The solid and shell elements have been connected by using the "Shell-to-solid" coupling option. The resulting FEM model of the wing is shown in Fig. 2.

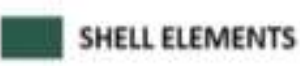

SOLID ELEMENTS

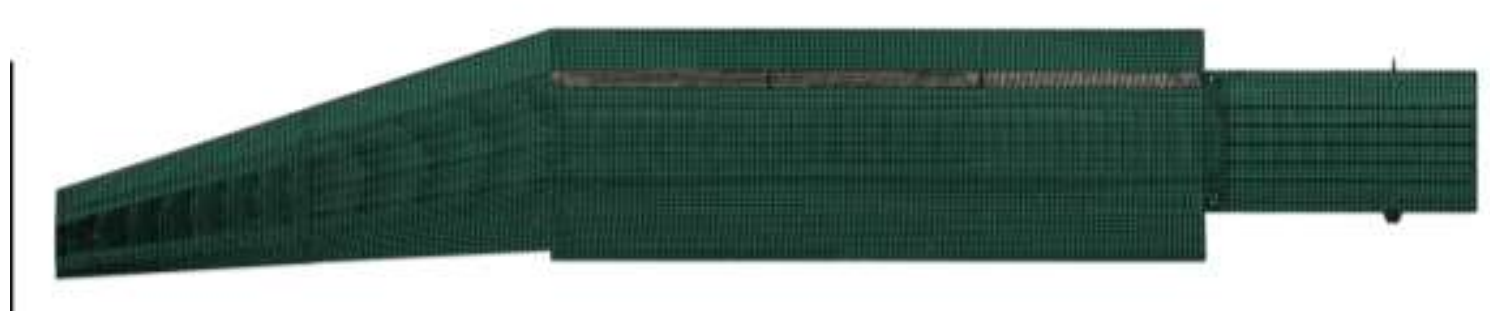

Fig. 2. FEM model.

Among all the detected defects, only the one shown in Fig. 3 has been considered relevant for the numerical analyses presented here. Indeed, due to its position along the wingspan and through the thickness, it is expected to propagate under service loading conditions. In order to simulate the delamination, tie constraints have been used between two sub-laminates, except in the delaminated area where contact elements have been placed to avoid penetrations. 


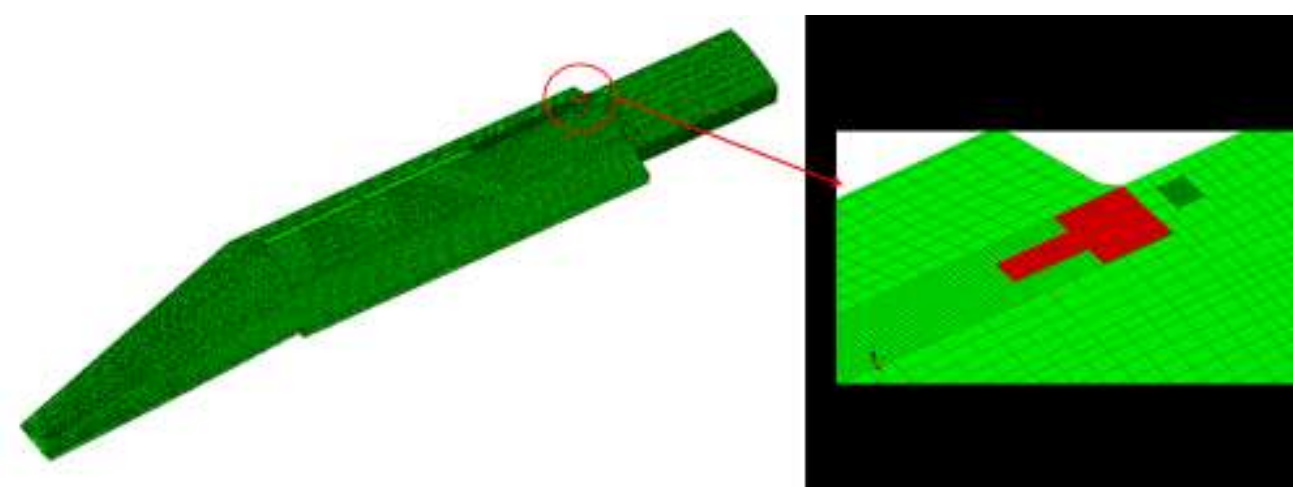

Fig. 3. Analysed delamination.

Two load conditions, related to symmetric and anti-symmetric manoeuvres have been considered for the analyses and reported in Table 2. The aerodynamic load distributions have been numerically simulated by applying equivalent concentrated forces at different stations along the wingspan.

\begin{tabular}{|c|c|c|}
\cline { 2 - 3 } \multicolumn{1}{c|}{} & LOAD CASE TYPE & LOAD CASE \\
\hline L1 & Symmetric Manoeuvres & Vc (Design cruising speed) in MTOW configuration \\
\hline L2 & $\begin{array}{c}\text { Conditions for Roll } \\
\text { Manoeuvres }\end{array}$ & $\begin{array}{c}\text { Partial aileron deflection with Va (Design manoeuvring } \\
\text { speed) in MTOW configuration }\end{array}$ \\
\hline
\end{tabular}

Table 2. Load conditions.

First, the global displacements of the structure have been evaluated by means of a non-linear static analysis. Secondly, linear buckling simulations have been carried out for each load condition to determine the instability load of the delamination. Finally, VCCT (Virtual Crack Closure Technique) analyses have been performed to assess the delamination propagation.

\section{Numerical results}

The deformed shapes with out-of-plane displacements contour plots for the load conditions L1 and L2 are respectively shown in Fig. 4 and Fig. 5.
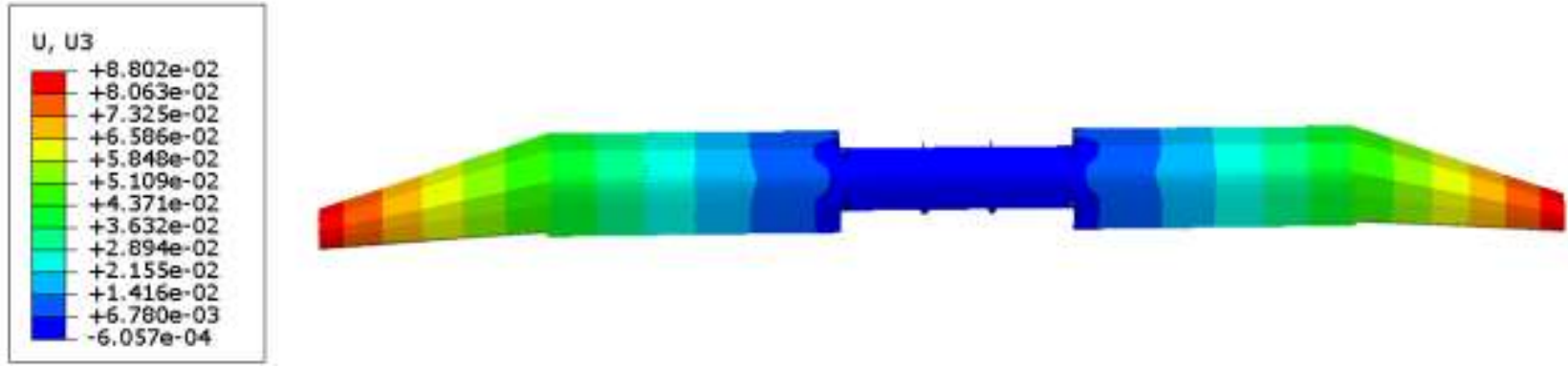

Fig. 4. Out-of-plane displacements - Load condition L1
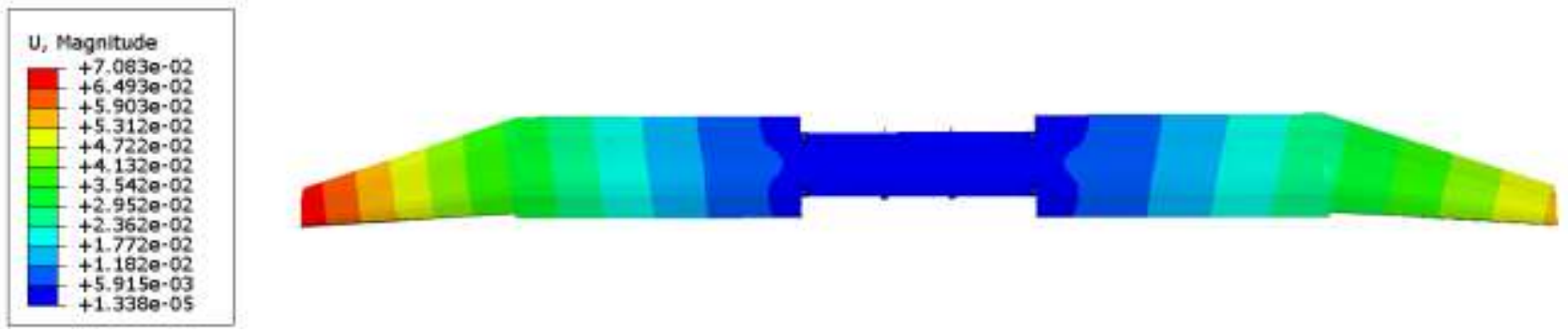

Fig. 5. Out-of-plane displacements - Load condition L2 
From the linear buckling analyses, it has been found that the critical load is over the service load for both loading conditions L1 (critical load is 1.32 times the limit load) and L2 (critical load is 1.71 times the limit load). The displacements contour plot of the linear buckling simulation for the load condition L1 is shown in Fig. 6(a), as an example. Because the delamination propagation onset is mostly affected by the local buckling of the defect, it should not propagate under service loads conditions. In order to verify this assumption, non-linear VCCT analyses have been performed. The numerical results have confirmed that no propagation takes place under the limit loading condition load for both loading conditions. The delamination front for the load condition L1 is shown, as an example, in Fig. 6(b), where the blue elements represent the delaminated area which does not increase during the VCCT analyses.

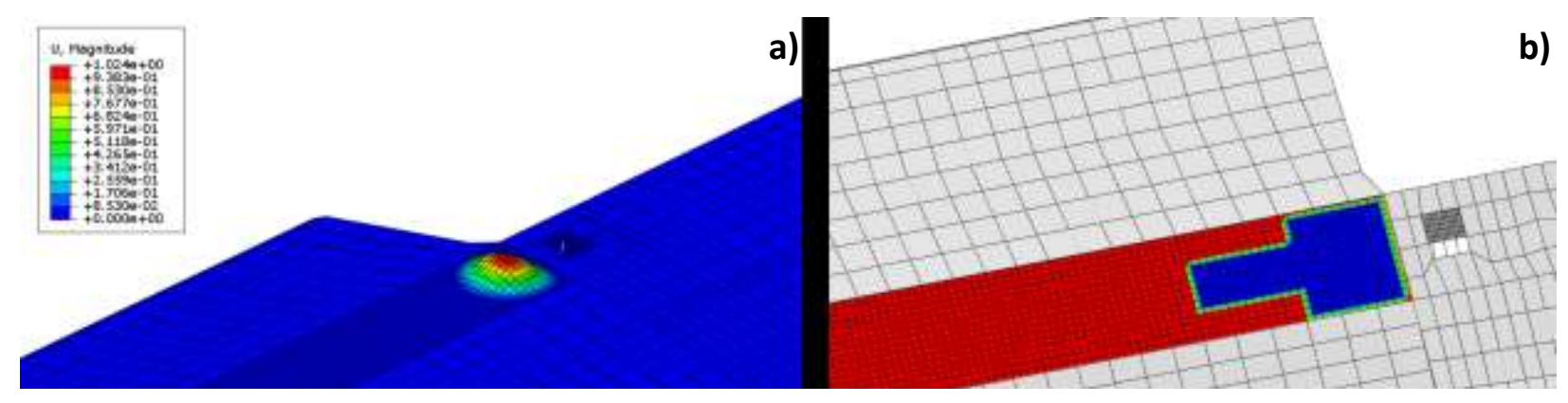

Fig. 6. (a) Delamination local buckling; (b) Delamination propagation - Load condition L1.

\section{Conclusion}

In this paper, the damage behaviour an all-composite wing has been studied. Indeed, the preexistent manufacturing induced damages elastic instability and propagation, under service loading conditions, have been assessed. The analyses have demonstrated that, in principle, the structure is damage tolerance and can withstand the service loading conditions.

\section{References}

[1] F. Caputo, G. Lamanna, A. De Luca, R. Borrelli and S. Franchitti. Global-Local FE Simulation of a Plate LVI Test; Structural Durability \& Health Monitoring - TechScience; (2013) 9 (3) 253267.

[2] R. Sepe, A. De Luca, G. Lamanna, F. Caputo, Numerical and experimental investigation of residual strength of a LVI damaged CFRP omega stiffened panel with a cut-out, Composites Part B: Engineering Journal; 102 (2016) 38-56.

[3] F. Caputo, G. Lamanna, A. De Luca, V. Lopresto. Numerical Simulation of LVI Test onto Composite Plates, Times of Polymers (TOP) and Composites 2014: Proceedings of the 7TH International Conference on Times of Polymers (TOP) and Composites; 1599, 334-337.

[4] F. Caputo, A. De Luca, G. Lamanna, R. Borrelli, U. Mercurio. Numerical Study for the Structural Analysis of Composite Laminates Subjected to Low Velocity Impact, Composite Part B: Engineering Journal; 67 (2014) 296-302.

[5] F. Xie, X. Wang, M. Li, Z. Zhang. Statistical Study of Delamination Area Distribution in Composite Components Fabricated by Autoclave Process. Applied Composite Materials (2009) 16(5): 285-295.

[6] A. Makeev, Y. Nikishkov, G. Seon, E. Armanios. Effects of defects on interlaminar performance of composites (Conference Paper). 39th European Rotorcraft Forum 2013, ERF 2013, Pages 354-364. 